\title{
Du Richard III de More à celui de Shakespeare : deux regards sur justice et pouvoir
}

\section{Marie-Claude Rousseau}

Marie-Thérèse Jones-Davies (éd.)

\section{(2) OpenEdition}

Journals

Édition électronique

URL : http://journals.openedition.org/shakespeare/1122

DOI : 10.4000/shakespeare.1122

ISSN : 2271-6424

Éditeur

Société Française Shakespeare

Édition imprimée

Date de publication : 1 novembre 1980

Pagination : $35-50$

Référence électronique

Marie-Claude Rousseau, «Du Richard III de More à celui de Shakespeare : deux regards sur justice et pouvoir », Actes des congrès de la Société française Shakespeare [En ligne], 2 | 1980, mis en ligne le 01 novembre 2007, consulté le 19 avril 2019. URL : http://journals.openedition.org/shakespeare/1122 ; DOI : 10.4000/shakespeare.1122 


\section{SOCIÉTÉ FRANC̣AISE SHAKESPEARE}

\section{ACTES DU CONGRĖS 1980}

DIRECTEUR DE LA PUBLICATION

M.T. Jones - Davies

JEAN TOUZOT Libraire - Editeur 38 , rue Saint-Sulpice 75278 PARIS CEDEX 061981 


\section{DU RICHARD III DE MORE A CELUI DE SHAKESPEARE : DEUX REGARDS SUR JUSTICE ET POUVOIR}

Qui est coupable ? De quoi ? Qui juge et qui condamne ? De quel droit ou en vertu de quel pouvoir? Aborder ces deux textes sur Richard III, dont l'un est la source principale de l'autre (puisque, pour sa pièce, Shakespeare s'est largement inspiré ', via Hall et Holinshed, du récit dramatique composé par More) c'est en quelque sorte instruire une affaire et tenter de répondre à des questions d'ordre juridique. Si aucun procès au sens strict du terme n'est mis en scène dans ces deux œuvres, chacune traite cependant de questions telles que le droit d'asile, le droit à la couronne, la légitimité ... Une thématique commune met en jeu les notions de Justice et Pouvoir et deux visions nous sont ainsi offertes : celle de More, homme de loi; celle de Shakespeare, homme de théâtre. L'analyse qui suit tend à souligner l'originalité des points de vue, en privilégiant celui de More, peut-être moins connu ${ }^{2}$.

Lorsque More entreprend l'histoire $\mathrm{du}$ roi Richard III ${ }^{3}$, texte bilingue (latin-anglais) composé entre 1513 et 1518 , c'est en juriste averti qu'il écrit. Issu d'une famille d'hommes de loi, fils de juge et petit-fils de sheriff, il a été formé dans ce haut lieu de l'enseignement du droit qu'étaient les Inns of Court de Londres, considérées alors comme la troisième université d'Angleterre. Le droit, il l'a lui-même enseigné, à Furnivall's Inn, puis à Lincoln's Inn où il fut par deux fois Reader (Autumn Reader en 1510 et Lent Reader en 1515 , une des plus hautes fonctions académiques dans la profession). Il le pratique enfin : admis au Barreau de Londres en 1501, il est à l'époque under-sheriff de la Cité, une tâche qui l'amène à administrer la loi, à siéger comme juge auprès du sheriff et à faire respecter l'ordre, comme lors des émeutes de mai 1517 . Il continuera de le pratiquer, au service du roi. L'image que la traditionl gardera de lui sera celle d'un avocat avisé et astucieux, d'un juge équitable, d'un Grand Chancelier efficace et intègre, «that worthy and uncorrupt magistrate» dont parlera Sir John Harrington soixante ans après son exécution. 
Rien d'étonnant donc à ce que More développe des questions de droit que Shakespeare se contentera d'aborder. La question du droit d'asile fait ainsi l'objet d'un véritable traité où il pèse le pour et le contre comme au tribunal. Il aime à signaler la compétence juridique de tel personnage, compétence parfois utilisée à de mauvaises fins comme dans le cas du perfide Catesby «which was a man well learned in the laws of this land». Il souligne l'importance de la rhétorique, parfois fallacieuse, telle cette plaidoirie de Buckingham («so evil a tale so well told») en contrepoint à l'éloquence d'un Rivers «a very well spoken man» 5 , victime innocente qui n'aura pas le loisir de plaider sa cause. Il se plaît à préciser des aspects de la juridiction anglaise, surtout dans son texte latin destiné au public Outre-Manche. Ce sont là, en marge d'un vocabulaire juridique plus abondant que chez Shakespeare, des notations d'expert. Voilà pour la forme; qu'en est-il du fond?

Le Richard III de More n'est pas, comme on l'a dit trop souvent, le sombre portrait moral d'un horrible tyran, assassin, seul responsable de tous les maux. Plus que d'une biographie, il s'agit ici d'une réflexion politique sur le pouvoir,à l'image de l'Utopic et des Epigrammes latines ${ }^{6}$, cuvres contemporaines. En tant que tel, en tant que réflexion sur la meilleure forme de gouvernement possible et sur les dangers qui menacent l'état, ce texte est une analyse : 1) de ce que devrait être un bon roi (dans l'idéal); 2) de ce qu'est un mauvais roi (danger toujours possible;Richard III déjà...); 3) du rôle à tenir auprès des princes sur la scène politique.

\section{L'IDEAL : la justice d'un roi-père, «sub Deo et sub lege» ${ }^{7}$}

Ce n'est pas sans raison que le récit de More s'ouvre par le portrait (idéalisé ?) d'Edouard IV . Le bon roi est un père pour son peuple, et non un maître. Il est le chien de berger qui protège le troupeau contre la venue du loup. (Le tyran, c'est le loup). Sa meilleure armure, sa meilleure protection ce ne sont pas ses palais fortifiés ni les insignes de sa royauté ${ }^{8}$. C'est l'amour que son peuple a pour lui. Nous pouvons opposer ici Shakespeare («the King's name is a tower of strength» V.3) à More (l'amour du peuple aurait 
¿û être pour les enfants d'Edouard «a marvelous fortress and sure armour») ${ }^{9}$. Le roi et son peuple ne font qu'un. Tel est aussi l'enseignement des Epigrammata : c'est le peuple qui, par son consentement, consacre le roi. En Utopie, le prince est même élu par la communauté. Peinture idéale ? Pas tant que cela. Edouard IV avait été couronné par acclamation populaire. Richard recherche lui aussi (c'est une constante dans le texte de More) l'assentiment du peuple. Un peuple, différent du troupeau de moutons apeurés peint par Shakespeare. Le seul éclair de lucidité qu'il aura dans la pièce de Shakespeare à propos du vice de forme de l'acte qui condamne Hastings ( Who is so gross/That cannot see this palpable devise ?»III.6) n'est qu'un pâle reflet de la clairvoyance quasi permanente des citoyens de Londres ( «not common people only, that wave with the wind, but wise men $)^{10}$ qui chez More sont des spectateurs, souvent impuissants, mais perspicaces, des coups montés par Richard et ses acolytes

Every man answred him fair, as though no man

mistrusted the man, which of truth no man believed ${ }^{11}$

there was no man so dull that heard them but he perceived well enough that all the matter was made between them.$^{\mathbf{2}}$

Chez More, le peuple est juge. Les citoyens opposent un silence réprobateur aux mises en scène de Richard

But the people were so far fro crying «King

Richard ${ }^{13}$ that they stood as they had been turned into stone

all was hushed and mute and not one word answered thereunto. ${ }^{14}$

Ils réagissent avec tristesse, «not able to dissemble their sorrow $\gg^{15}$ ou avec humour, un humour parfois mordant, comme dans le cas du «shameful sermon»sur la légitimité de Richard prononcé par un certain Shaw qui n'osa plus après sa désastreuse prestation mettre le nez dehors «but kept him out of sight like an owl $\rangle^{16}$ et finit par en mourir de honte («within few days after, he withered and consumed away $)^{17}$. Clin d'œil de l'humoriste : il y a tout de même une justice dans ce bas monde !

More avait une haute estime du service de la Cité. Le portrait élogieux qu'il donne de cette dernière est l'hommage d'un de ses 
fonctionnaires à un peuple qu'il aimait et qui l'aimait. Peutêtre faut-il voir ici une distinction entre la vision de Shakespeare, country gentleman de Stratford et More, enfant chéri de la Cité de Londres

«the best friend that the poor e'er had»

comme l'a immortalisé la pièce élisabéthaine Sir Thomas More. Il est vrai que More défendra souvent le peuple. Il se dressera contre Wolsey et réclamera la liberté d'expression aux Communes en 1523. Et Alison Hanham n'hésite pas à voir en More le premier historien anglais à avoir donné au peuple la place qui lui est due ${ }^{18}$.

Le roi c'est aussi celui qui administre la loi, d'où la nécessité pour lui d'être entouré par de bon conseillers qui lui inspireront «des mesures conformes au droit et à la morale». Le bien public en dépend :

«C'est du prince, en effet, que découle, comme d'une source intarissable, le torrent des bienfaits et des maux qui abreuvent tout un peuple.» «s'il est vrai que le salut ou la ruine de la communauté politique dépendent de la conduite de ceux qui exercent l'autorité et la justice ${ }^{19}$

Exercice de la loi et royauté sont donc étroitement liés. Richard, dans le récit de More, l'a bien compris lui qui, le 26 juin 1485 arrive à Westminster Hall en grande pompe (scène qui correspond chez Shakespeare à IV.2)

«and there, when he had placed himself in the Court of the King's Bench, declared to the audience that he would take upon him the crown in that place there, where the king himself sitteth and ministreth the law, because he considered that it was the chiefest duty of a king to minister the laws.» 20

Historiquement, Richard III data d'ailleurs officiellement son règne à compter de ce jour où il s'assit publiquement au Banc du Roi.

Le devoir du roi est donc de faire respecter la loi. Ainsi le prince Edouard fut-il envoyé au pays de Galles «Which country, being far off from the law and 
recourse to justice, was begun to be far out of good will (...). And for this encheason the prince was in the life of his father sent thither, to the end that the authority of his presence should refrain evil disposed persons fro the boldness of their former outrages; ${ }^{21}$

Mais s'il est garant de la loi, le roi n'est pas pour autant audessus d'elle. Bien au contraire. Il est, selon l'expression de Bracton, «sub Deo et sub lege», «under God and the law», conception primordiale chez More qui se situe ainsi dans la tradition médiévale pour séparer droit divin ou loi divine d'une part et pouvoir royal d'autre part, ce qui l'amène dans son Utopie à remettre en cause l'axiome de droit public anglais selon lequel «the king can do no wrong». Le roi est tenu de respecter «the law of God», «the law of nature», «the law of the land», une argumentation qu'il développe à propos du droit d'asile dans Richard III. Une telle conception, qui lui vaudra d'ailleurs d'être décapité, s'oppose radicalement à l'image Tudor de la monarchie de droit divin avec son corollaire : la rébellion est un crime contre Dieu,héritage de Tyndale,véhiculé par les IIomelies et repris par Shakespeare. De ce point de vue, le discours de More lors des insurrections de mai 1517, texte attribué à Shakespeare dans la pièce élisabéthaine Sir Thomas More, ressemble peut-être beaucoup au discours d'Ulysse dans Troilus and Cressida mais assez peu à More.

Le peuple enfin n'a pas besoin, selon More, d'un prince vertueux, au sens de chaste. Au roi Edouard, quelque peu licencieux, il ne tient pas rigueur mais il ne peut souffrir les méfaits de Richard qui, lui, joue au redresseur de torts et oblige la femme de Shore,maitresse du roi avant d'être celle d'Hastings, à faire pénitence publique dans les rues de Londres, le corps à peine vêtu. Il la condamne

«as a goodly continent prince, clean and faultless of himself, sent out of heaven into this vicious world for the amendment of men's manners"

Une fois de plus, le peuple est bon juge. Le plus grand crime n'est pas péché de la chair ( «fleshy wantonness»); le temps, c'est-à-dire la vieillesse, en sera le meilleur justicier ${ }^{23}$ Et en voyant passer Mrs. Shore, les citoyens seront finalement 
plus amoureux de son corps que soucieux de son âme «more amorous of her body than curious of her soul !»

Le véritable danger qui menace l'Etat, c'est un autre appétit. L'appétit du pouvoir.

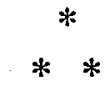

LES DANGERS QUI MENACENT L'ETAT : «painted process»; «lawless enterprises»

Le tyran, c'est donc le loup affamé de pouvoir. Le roi n'est plus alors «le gardien d'un royaume, mais d'une prison» (O Hamlet !...). L'appétit du pouvoir («the execrable desire of sovereignty»), l'ambition («a pestilent serpent»), la jalousie conduisent à la haine, à la guerre civile, au mépris de tous les liens

«all the bands broken that binden man and man together, without any respect of God or the world ${ }^{24}$.

De tels maux mettent en danger la communauté

«Dès que ces deux maux, partialité et cupidité, interviennent dans les jugements, c'en est fait, sur le champ, de toute la justice, le nerf le plus puissant de l'Etat.» ${ }^{25}$

C'est la porte ouverte, en matière de justice, à la flatterie : More fustige la flagornerie des hommes de loi qui tournent toutes les causes, fût-ce les plus mauvaises, à l'avantage du roi. Richard essaie ainsi de se concilier les bonnes grâces des citoyens, «but specially the lawyers of this realm ${ }^{26}{ }^{6} \mathrm{C}$ 'est la porte ouverte à l'arbitraire. On condamne sans jugement, ce qui est la négation même des principes émis par la Magna Carta: Rivers, Vaughan et Grey sont décapités «without judgement, process or manner of order» et chez Shakespeare Clarence s'étonne pareillement : «who pronounced/The bitter sentence of poor Clarence's death/ Before I be convict by course of law ?» (I.4). Très vite, la force remplace alors la loi sur un terrain où le droit s'opposait déjà à l'équité. Le tyran trouve dans la vanité et l'ambition des hommes une base sur laquelle il s'appuie ${ }^{27}$. 
La loi est détournée à des fins injustes et n'est plus qu'une parodie de justice.

L'idéal est que loi et équité se rejoignent. More,dans l'exercice de ses fonctions, notamment comme Grand Chancelier, s'efforcera d'en réaliser la réconciliation. En Utopie, point n'est besoin de lois compliquées puisque chaque citoyen est vertueux et juste. Budé dans sa lettre à Lupset, qui sert de préface à l'Utopie, définit celle-ci comme Hagnopolis, c'est-à-dire non seulement la Cité des Justes, mais celle des Innocents. More met donc la notion de justice considérée comme vertu au-dessus de la justice : réglementation, droit codifié. Mieux vaut être vertueux qu'expert en droit; on ne gagne pas toujours à être plus instruit que foncièrement juste : tel est le cas du pauvre Shaw, précédemment cité, homme «of great learning, but of less virtue than learning ${ }^{28}$

La véritable justice est souvent étouffée sous un fatras de termes juridiques que manipulent des magistrats, pratiquant «l'art de feindre» (ce que More qualifie de «painted process» dans son Richard III, un art alors exercé par le tyran lui-même). De là à justifier la force par la loi, il n'y a qu'un pas. La loi peut être un masque derrière lequel se dissimule le vice, «sous couvert du droit» ${ }^{29}$ (《iustitiae colore», «praetextu juris»). La justice est alors inique et la tyrannie, selon More, c'est cela : «lawless enterprises ${ }^{30} \mathrm{ou}$ selon le latin, «tyrannica facinora».

Si Richard, dans le récit de More, est coupable (et avec lui, Catesby, Buckingham, Hastings, Rivers et même Clarence, etc.) ce n'est pas, en premier chef, d'être un virtuose du crime, c'est d'avoir détourné la loi; c'est d'avoir détruit les bases de la justice naturelle. Son plus grand crime est finalement d'avoir pris le masque de la légalité pour abuser le peuple, et cela (circonstances attenuantes ?) en s'appuyant sur l'ambition politique des autres. Ses juges ? Les citoyens de Londres. Richard essaie de les duper par une parodie d'élection populaire à Baynard's Castle mais ce n'est qu'une mascarade et un couronnement de comédie :

«after this mockish election, then was he crowned the sixth of july. (...) Now fell their mischief thick. And as the thing evil gotten is never well kept, through all the time of his reign never ceased there cruel death and slaughter, till his own 
destruction ended it.» ${ }^{31}$

Richard est condamné, avant même le récit du meurtre des petits princes.

Plus que l'ambition, ce que la pièce de Shakespeare met en cause, c'est la violence. Le meurtre appelle le meurtre; le sang est versé pour prix du sang. Chez More, on l'a vu, l'ambition est ce serpent venimeux qui, une fois entré dans le cœur des hommes, tourne tout en mal.

«... But I am in

So far in blood that sin will pluck on sin»(IV.ii).

La culpabilité, chez Shakespeare, est d'un autre ordre : elle a la couleur du sang. En dehors des petits princes, toutes les victimes de Richard sont elle-mêmes coupables de meurtre; Magaret se charge de le leur rappeler. More, lui, laisse planer le doute sur de telles responsabilités, y compris celle de Richard dans l'assassinat de Clarence et même dans celui des enfants d Edouard

"whose death and final infortune hath nevertheless so far comen in question that some remain yet in doubt whether they were in his days destroyed or no».32

Chez More, ce qui perd les uns, c'est leur soif du pouvoir (Edouard, Clarence et Richard étaient tous les trois «greedy and ambitious of authority»; Buckingham crevait de jalousie à la vue de la couronne sur la tête de Richard). Ce qui perd les autres c'est leur naïveté face à la ruse du tyran, «a deep dissimuler» (ainsi Hastings, «trusty enough, trusting too much»).

Dans la pièce de Shakespeare, «Bloody thou art, bloody will be thy end» (IV.iv) est la conclusion logique d'un mécanisme de la violence. Jusqu'à ce que s'abatte le couperet de la justice

«Thus hath the course of justice whirled about» (IV.iv).

Quelle justice? Celle de Dieu :

«Thou wilt die by God's just ordinance»(IV.iv). Richmond n'en sera que l'instrument. Aux Assises de Dieu, Richard est condamné. Ironie tragique : il est d'abord le justicier de tous les autres coupables. La pièce tout entière est une condamnation liturgique dont il est, pour finir, le bouc émissaire. Les fantômes de ses victimes, témoins à charge, le convoquent à la barre : 
"All several sins, all used in each degree, throng to the bar, crying all «Guilty!Guilty!»» (V.iii).

Et la sentence tombe, inéluctable : «Despair and die».

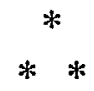

THEATRE ET ECHAFAUD. LA VIOLENCE ET

LE SACRE' : «Kings' games»; «stage plays»

Les planches du théâtre et celles de l'échafaud où la victime, innocente ou coupable, est immolée, sont bien souvent du même bois. Liturgie sacrificielle du théâtre, certes; plus applicable au texte de Shakespeare qu'à celui de More donc. Mais aussi réflexion sur le jeu politique et ce qu'il en coûte de monter sur la scène auprès des princes.

«And in a stage play all the people know right well that he that playeth the sowdaine is percase a sowter. Yet if one should can so little good to show out of season what acquaintance he hath with him and call him by his own name while he standeth in his majesty, one of his tormentors might hap to break his head, and worthy, for marring of the play. And so they said that these matters be king's games, as it were, stage plays, and for the more part played upon scaffolds, in which poor men be but the lookers-on. And they that wise be will meddle no farther. For they that sometime step up and play with them, when they cannot play their parts, they disorder the play and do themself no good $\gg .{ }^{34}$

Tel est donc le point culminant du texte de More, le sommet de la réflexion politique qu'il poursuit dans cette biographie de Richard III. Prendre le pouvoir, c'est monter sur une scène de théâtre en quelque sorte. Ce sont là jeux de rois; des jeux dangereux pour ceux qui se risquent à y monter auprès d'eux. Autant jouer avec des lions en cage nous dit-il dans un de ses poèmes latins, ${ }^{35}$ ce qui n'est pas sans évoquer cet avertissement de Margaret à Buckingham : «Look when he fawns he bites; and when he bites His venom tooth will rankle to the death».(I.3) 
Le jeu politique est finalement un jeu de chat et de souris : seuls les plus malins s'en tirent (ainsi de Morton comparativement à Hastings, trop crédule, ou à Buckingham, incapable de louvoyer plus longtemps).

Il peut paraître paradoxal que ce soit More, l'homme de loi, qui nous fournisse cette comparaison entre théâtre et échafaud, théâtre et pouvoir, théâtre et justice. Rien d'étonnant à cela pourtant puisque More, rappelons-le, avait été formé dans les Inns of Court célèbres pour leurs Revels (le père de More avait été Master of the Revels quand le jeune Thomas avait neuf ou dix ans et il étonnait les invités de Morton, chez qui il était page, èn montant sur la scène pour se mêler aux acteurs); Erasme prétend même que More composa des pièces de théâtre. Toujours est-il que dans son environnement proche (John Rastell, son beaufrère, avait un théâtre dans sa propriété et participa plus d'une fois à l'organisation de pageants), le théâtre était une réalité presque aussi vivante que le droit. C'est donc le fruit d'une expérience directe qu'il nous livre, aussi bien avec le théâtre qu'avec l'exercice de la loi ou du jeu politique.

Or sur cette scène politique, Richard a joué et perdu. Chez More, il perd pour deux raisons : parce que s'il est un roi-acteur «a player king», il est un acteur râté. Tous ces coups montés échouent lamentablement, au moins aux yeux du peuple, faute de synchronisation dramatique : Shaw fait son sermon à contretemps «out of all order and out of all frame»; l'acte d'accusation d'Hastings est proclamé beaucoup trop tôt

«Here is a gay goodly cast, foul cast away for haste.$^{36}$

Quant à la prise du pouvoir par Richard, elle est une mise en scène si évidente que le peuple en voit aisément l'artifice. C'est la deuxième raison de l'échec de Richard; nous l'avons déjà signalé : on ne revêt pas impunément le masque de la légalité pour abuser le peuple.

Le Richard III de Shakespeare est, lui, avant tout prisonnier de son rôle de roi, un rôle qu'il a usurpé il est vrai. Il semble que ce rôle lui colle à la peau. Il est à noter, me semble-t-il, que dans cette pièce (si nous en étudions la structure propre sans faire référence à Itenry VI, part III) 
Richard n'explicite pas son désir d'être roi avant de l'être devenu ( Am I king ?»IV.ii) et encore l'interrogation laisse percer le doute sur le rôle exact à tenir. Son intention est bien au départ de tenir le rôle du villain ou du diable «I am determined to prove a villain» (I.i)

«And seem a saint, when most I play the devil»

(I. iii).

Or tout à coup il découvre qu'il est vraiment un villain; entre-temps, de bouffon il est devenu roi et assassin «ls there a murderer here? No. Yes, I am» «I am a villain» (V. iii)

Son visage, prisonnier du masque sacré de roi («the Lord's anointed» comme il le dit lui-même), ne peut être mis à nu que dans un déchirement, violent, tragique. La «violence et le sacré ${ }^{37}$. Un déchirement qui préfigure celui de Macbeth de Lear et de Hamlet.

Richard doit payer, car là était le crime : avoir usurpé le masque sacré đe la royauté pour finalement n'abuser que lui-même.

«My kingdom for a horse!»

Richard, soldat égaré sur la scène politique, n'était pas fait pour les jeux de cour ( laquelle d'ailleurs : celle du roi ou celle du tribunal ? Pouvoir et justice se recoupent une fois de plus). Il n'a plus que mépris pour les mots («words») qu'il n'a pas su maîtriser et leur préfère l'épée de jadis («swords»), somme toute la force à la loi

«Conscience is but a word that cowards use, devised at first to keep the strong in awe :

Our strong arms be our conscience, swords our

law»(V.iii).

Son jeu à lui va à l'encontre de toutes les règles. Son dernier cri est celui d'un solitaire, ayant misé sa vie sur un coup de dé,

«Slave, I have set my life upon a cast,

And I will stand the hazard of the die.»(V.iv)

Mais le droit l'emportera sur la force. Ou plus exactement une autre force. Il faut en effet que Richard soit coupable pour que soient purgées toutes les transgressions. Théâtre, justice et sacrifice se confondent. Toutes les sociétés de réhabilitation de la cause de Richard n'y pourront rien.Peu importe que More soit coupable d'avoir donné naissance ou mythe du tyran. Peu importe que Shakespeare l'ait noirci 
encore. Au théâtre et surtout dans l'inconscient collectif, il faut que Richard soit coupable. «A horse ! A horse ! My kingdom for a horse !» (V.4) Exit Richard.

$\mathrm{Au}$ tribunal de l'Histoire, son plus grand crime est en définitive d'avoir perdu la bataille de Bosworth. Car s'il avait gagné ...

«It is perilous striving with princes» ${ }^{38}$. Ces mots de Norfolk à More seront un avertissement. Quelques pages après le passage où il réfléchit sur le jeu politique, More laisse son œuvre inachevée. Simple prudence, peut-être : les hommes au pouvoir se suivent et se ressemblent; More n'at-il pas dénoncé en 1509 les agissements du successeur de

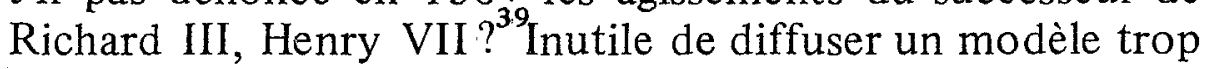
facilement imitable. Et pourtant au même moment, il abandonne le service de la Cité pour celui du roi, dans l'espoir de tenir sans doute auprès de lui le rôle de «philosopheconseiller» qu'il recommande dans son Utopie. Il semble cependant conscient du danger

«I may tell thee I have no cause to be proud threof, for if my head could win a castle in France, It should not fail to go.»

dit-il à Roper ${ }^{40}$. On connaît la suite. En 1532 il démissionne et en 1535, il est jugé, condamné à mort et exécuté. Il meurt d'avoir voulu, comme Beckett, préférer l'honneur de Dieu au pouvoir du roi. Il s'est voulu finalement citoyen d'un autre royaume. Il se sent plus libre, prisonnier dans la Tour de Londres que Richard, prisonnier de son rôle de roi sur le champ de bataille de Bosworth. Son procès et son exécution marqueront un changement dans l'histoire du droit anglais ${ }^{41}$. Il oppose au pouvoir du roi, son silence, fort $\mathrm{du}$ droit de sa conscience et de la loi divine

«for this my taciturnity and silence, neither your law nor any law in the world is able justly and rightly to punish me» 42

«and therefore am I not bounden, my Lord, to conform my conscience to the Council of one realm against the general Council of Christendom.» 43

Ses derniers mots sur l'échafaud : «I die the King's good servant, but God's first» résument sa conception de la justice (naturelle ou divine) et du pouvoir temporel. 


\section{Exit Thomas More «A very learned worthy genteman}

Seals error with his blood. Come, we'll to Court.»

Telle est la conclusion de la pièce élisabéthaine Sir Thomas More, peut-être entièrement de Shakespeare. Mais ceci est une autre affaire ...

\section{Marie-Claude ROUSSEAU}

\section{NOTES}

1. Le texte de More couvre la pièce de Shakespeare jusqu'à IV.4, c'est-à-dire à la veille de la rébellion de Buckingham.

2. «More the lawyer is perhaps even less studied than More the saint and More the student of the sacred siences." R.J. Schoeck in «Sir Thomas More, humanist and lawyer», University of Toronto Quarterly, 26 (1964) 1-14, reproduit in Essential Articles for the Study of St. Thomas More, ed. Richard S. Sylvester et Germain Marc'hadour (Hamden, Connecticut, 1977) 569-582. Un essai de bilan critique et biliographique sur More et le droit est présenté par R.J. Schoeck in "The place of Sir Thomas More in legal history and tradition : a sketch with some observations», Moreana, 51 (1976) 83-94. Pour un éclairage différent et plus récent, voir J.A. Guy, The Public Career of Sir Thomas More (Brighton: The Harvester-Press, 1980). Voir aussi sur cette question les essais de Margaret Hastings et/de Richard O'Sullivan in Essential Articles.

Il reste qu'aucune étude systématique de l'aspect juridique du $R$ ichard $I I I$ de More n'a été publiée à ce jour.

3. La meilleure édition est celle établie par R.S.Sylvester : Complete Works of St. Thomas More, vol.2 ( Yale Univ. Press, 1963). Sylvester en a publié depuis une édition simplifiée, où l'orthographe et la ponctuation ont été modernisées : The History of King Richard III and selections from the English and Latin Poems (Yale Univ. Press, 1976). C'est à celle-ci que nous nous rapportons pour nos citations, mentionnées sous le nom de Sylvester.

4. "When More some time had Chancellor been/No more suits did remain;/The like will never more be seen/ Till More be there again." in John Campbell, Lives of the Chancellors (1849), I, 551.

5. Un trait que Shakespeare reprend à propos de Clarence; Richard aux assassins : «donot hear him plead;/ For Clarence is well-spoken»(I.iii).Sur Catesby et Buckingham, voir Sylvester ed. pp. 46 et 77.

6. Les Epigrammata, poèmes latins composés entre 1497 et 1517 et publiés en 1518 ont été répertoriés et traduits en anglais par L. Bradner et C.A. Lynch : The Latin Epigrams of Thomas More (Univ. of Chicago Press, 1953). L'introduction précise : «Among these topics, the most original for an epigrammatist was Kingship.In fact, we know of no other sixteenth-century poet who used this theme for short poems.» p. xxxii . Les poèmes Nos 227, 91, 92, 
$94,96,97,102,103,144,182$ sont particulièrement utiles pour le point de vue qui nous intéresse.

Pour l'Utopie, nous utilisons l'édition d'André Prévost (Mama,1978) citée sous le nom de Prévost.

7. Bracton, De Legibus, cité par Richard O'Sullivan ed. en exergue à Under God and the Law (Oxford, 1949).

8. Epigrammata, No $91,97,102$. cf. Sylvester 0.25 «the lamb was betaken to the wolf» .

9. Sylvester ed. p.6

10. Ibid. p. 45

11. Ibid. p. 53

12. Ibid. p. 82

13. Ibid. p. 69

14. Ibid. p. 76

15. Ibid. p.79

16. Ibid. p.69

17. Ibid. p.69 p. 185 .

18. in Richard III. and his Early Historians 1483-1535 (Oxford, 1975)

19. trad. Prévost, p. 374

20. Sylvester ed. p. 83

21. Ibid. p. 14

22. Ibid. p. 55

23. Ibid. p. 5 à propos d'Edouard IV et p.56 au sujet de Mrs. Shore

24. Ibid. p. 6

25. trad. Prévost p. 562

26. Sylvester ed. p. 83

27. Ibid. p. 10 «a sure ground for the foundation of all his building».

28. Ibid. p. 59

29. trad. Prévost p. 569; "painted process» in Sylvester ed. p. 38

30. Sylvester ed. p. 58 
31. Ibid. p. 84

32. Ibid. p. 84

33. Ibid. p. 53

34. Ibid. p. 83

35. Epigrammata, No 144

36. Ibid. p. 55

37. allusion au livre de René Girard, La Violence et le sacré (Grasset, 1972).

38. rapporté par Chambers, Thomas More (London, 1935) p. 300

39. dans son poème composé en 1509 à l'occasion du couronnement d'Henry VIII il dénonce «even laws put to unjust ends» sous le règne d'Henry VII.

40. Roper, Life of More in Two Early Tudor Lives, ed. R.S. Sylvester et D.P. Harding (New Haven, 1962) p.208

41. Richard O'Sullivan, The King's Good Servant : papers read to the Thomas More Society of London (1948) p. $7:$ "The death of Sir Thomas More may fairly be said to mark a turning-point in English law and history».

42. Reynolds, Saint Thomas More (London, 1953) p. 342

43. Chambers, op. cit. p. 341 